\title{
Front Matter: Volume 7940
}

, "Front Matter: Volume 7940," Proc. SPIE 7940, Oxide-based Materials and Devices II, 794001 (7 April 2011); doi: 10.1117/12.891665

SPIE. Event: SPIE OPTO, 2011, San Francisco, California, United States 


\title{
PROCEEDINGS OF SPIE
}

\section{Oxide-based Materials and Devices II}

\author{
Ferechteh Hosseini Teherani \\ David C. Look \\ David J. Rogers \\ Editors
}

23-26 January 2011

San Francisco, California, United States

Sponsored and Published by

SPIE 
The papers included in this volume were part of the technical conference cited on the cover and title page. Papers were selected and subject to review by the editors and conference program committee. Some conference presentations may not be available for publication. The papers published in these proceedings reflect the work and thoughts of the authors and are published herein as submitted. The publisher is not responsible for the validity of the information or for any outcomes resulting from reliance thereon.

Please use the following format to cite material from this book:

Author(s), "Title of Paper," in Oxide-based Materials and Devices II, edited by Ferechteh Hosseini Teherani, David C. Look, David J. Rogers, Proceedings of SPIE Vol. 7940 (SPIE, Bellingham, WA, 2011) Article CID Number.

ISSN 0277-786X

ISBN 9780819484772

Published by

SPIE

P.O. Box 10, Bellingham, Washington 98227-0010 USA

Telephone +1 3606763290 (Pacific Time) · Fax +1 3606471445

SPIE.org

Copyright (C) 2011, Society of Photo-Optical Instrumentation Engineers

Copying of material in this book for internal or personal use, or for the internal or personal use of specific clients, beyond the fair use provisions granted by the U.S. Copyright Law is authorized by SPIE subject to payment of copying fees. The Transactional Reporting Service base fee for this volume is $\$ 18.00$ per article (or portion thereof), which should be paid directly to the Copyright Clearance Center (CCC), 222 Rosewood Drive, Danvers, MA 01923. Payment may also be made electronically through CCC Online at copyright.com. Other copying for republication, resale, advertising or promotion, or any form of systematic or multiple reproduction of any material in this book is prohibited except with permission in writing from the publisher. The CCC fee code is $0277-786 \mathrm{X} / 11 / \$ 18.00$.

Printed in the United States of America.

Publication of record for individual papers is online in the SPIE Digital Library.

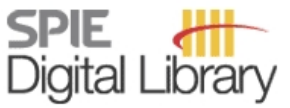

SPIEDigitalLibrary.org

Paper Numbering: Proceedings of SPIE follow an e-First publication model, with papers published first online and then in print and on CD-ROM. Papers are published as they are submitted and meet publication criteria. A unique, consistent, permanent citation identifier (CID) number is assigned to each article at the time of the first publication. Utilization of CIDs allows articles to be fully citable as soon as they are published online, and connects the same identifier to all online, print, and electronic versions of the publication. SPIE uses a six-digit CID article numbering system in which:

- The first four digits correspond to the SPIE volume number.

- The last two digits indicate publication order within the volume using a Base 36 numbering system employing both numerals and letters. These two-number sets start with 00, 01, 02, 03, 04, $05,06,07,08,09,0 A, 0 B \ldots 0 Z$, followed by 10-1Z, 20-2Z, etc.

The CID number appears on each page of the manuscript. The complete citation is used on the first page, and an abbreviated version on subsequent pages. Numbers in the index correspond to the last two digits of the six-digit CID number. 


\section{Contents}

ix Conference Committee

ZNO DOPING

794003 Donor and acceptor concentrations from a single mobility measurement in degenerate semiconductors: ZnO [7940-02]

D. C. Look, Wright State Univ. (United States) and Air Force Research Lab. (United States);

R. C. Scott, Arizona State Univ. (United States); K. D. Leedy, B. Bayraktaroglu, Air Force Research Lab. (United States)

794006 Photoluminescence and hall study for the production and recovery of defects in phosphorus implanted ZnO films [7940-05]

S. Nagar, B. Sinha, A. Mandal, S. Chakrabarti, Indian Institute of Technology, Bombay (India);

S. K. Gupta, Bhabha Atomic Research Ctr. (India)

ZNO NANOSTRUCTURES: GROWTH AND DEVICE FABRICATION I

794009 Zinc oxide nanostructures with metal particles based on surface plasmons for optoelectronic device applications (Invited Paper) [7940-08]

J. S. Yu, Y. H. Ko, H. K. Lee, J. W. Leem, Kyung Hee Univ. (Korea, Republic of)

7940 OA Intrinsic white-light emission from zinc oxide nanorods heterojunctions on large-area substrates (Invited Paper) [7940-09]

M. Willander, O. Nur, S. Zaman, A. Zainelabdin, G. Amin, J. R. Sadaf, M. Q. Israr, N. Bano,

I. Hussain, N. H. Alvi, Linköping Univ. (Sweden)

7940 OB ZnO nanorods for light-emitting diode applications (Invited Paper) [7940-10]

X. Chen, A. M. C. Ng, K. K. Wong, A. B. Djurišić, F. Fang, W. K. Chan, The Univ. of Hong Kong (Hong Kong, China); P. W. K. Fong, H. F. Lui, C. Surya, Hong Kong Polytechnic Univ. (Hong Kong, China)

7940 OC Synthesis and characterization of layer structured ZnO nanowire for ultraviolet light-emitting diode [7940-12]

D. Nakamura, A. Kumeda, K. Toya, K. Okazaki, K. Kubo, K. Tsuta, M. Higashihata, T. Okada, Kyushu Univ. (Japan)

\section{ZNO NANOSTRUCTURES: GROWTH AND DEVICE FABRICATION II}

7940 OE Bridging wide bandgap nanowires for ultraviolet light detection [7940-13]

Y. Li, M. Zhong, T. Tokizono, A. Paulsen, The Univ. of Tokyo (Japan); M. Liao, Y. Koide, National Institute for Materials Science (Japan); I. Yamada, J.-J. Delaunay, The Univ. of Tokyo (Japan) 
7940 OF Applications of zinc oxide nanowires for bio-photonics and bio-electronics (Invited Paper) [7940-15]

M. Willander, O. Nur, M. Fakhar-e-Alam, J. R. Sadaf, M. Q. Israr, K. Sultana, S. M. U. Ali,

M. H. Asif, Linköping Univ. (Sweden)

7940 OG Multifunctional ZnO nanostructures: from material growth to novel applications (Invited Paper) [7940-16]

X. Wang, J. Shi, Univ. of Wisconsin-Madison (United States)

TOWARDS UNDERSTANDING OXIDES PROPERTIES I

$794000 \quad$ A density functional theory study on the electronic and magnetic properties of (Mn,N)-codoped ZnO [7940-18]

L. Zhao, P. F. LU, Z. Y. YU, X. T. Guo, Y. Shen, H. Ye, G. F. Yuan, Beijing Univ. of Posts and Telecommunications (China)

7940 OJ Role of $\mathrm{Yb}^{3+}$ ions in the IR to visible upconversion of $\mathrm{Er}^{3+}$ ions in LTT glasses [7940-19] A. Mohan Babu, J. Suresh Kumar, Sri Venkateswara Univ. (India); B. C. Jamalaiah, Pukyong National Univ. (Korea, Republic of); N. K. Giri, S. B. Rai, Banaras Hindu Univ. (India);

L. Rama Moorthy, Sri Venkateswara Univ. (India)

7940 OK Thiol adsorption on metal oxides: an approach for selective deposition on zinc oxide nanoparticles [7940-20]

J. W. Soares, D. M. Steeves, U.S. Army Natick Soldier Research, Development and Engineering Ctr. (United States); J. Singh, J. Im, J. E. Whitten, Univ. of Massachusetts Lowell (United States)

$7940 \mathrm{OL} \quad$ Studies of PLD-grown ZnO and MBE-grown GaP mosaic thin films by $x$-ray scattering methods: beyond the restrictive $\omega$ rocking curve linewidth as a figure-of-merit (Invited Paper) [7940-21]

O. Durand, A. Letoublon, Univ. Européenne de Bretagne, INSA, FOTON, UMR (France); D. J. Rogers, F. H. Teherani, Nanovation SARL (France); C. Cornet, A. Le Corre, Univ. Européenne de Bretagne, INSA, FOTON, UMR (France)

\section{TOWARDS UNDERSTANDING OXIDES PROPERTIES II}

$7940 \mathrm{ON}$ Electronic structure, doping, and lattice dynamics of $\mathrm{LiGaO}_{2}$ (Invited Paper) [7940-23]

A. Boonchun, W. R. L. Lambrecht, Case Western Reserve Univ. (United States)

794000 Radiation damage formation and annealing in GaN and ZnO (Invited Paper) [7940-24]

K. Lorenz, Instituto Tecnológico e Nuclear (Portugal) and Univ. de Lisboa (Portugal); M. Peres, Univ. de Aveiro (Portugal); N. Franco, J. G. Marques, Instituto Tecnológico e Nuclear (Portugal) and Univ. de Lisboa (Portugal); S. M. C. Miranda, Instituto Tecnológico e Nuclear (Portugal); S. Magalhães, Instituto Tecnológico e Nuclear (Portugal) and Univ. de Aveiro (Portugal); T. Monteiro, Univ. de Aveiro (Portugal); W. Wesch, Friedrich-Schiller-Univ. Jena (Germany); E. Alves, Instituto Tecnológico e Nuclear (Portugal) and Univ. de Lisboa (Portugal); E. Wendler, Friedrich-Schiller-Univ. Jena (Germany) 
$7940 \mathrm{OQ}$ Emergence of superconductivity on a $\mathrm{SrTiO}_{3}$ surface by electric-field charge accumulation (Invited Paper) [7940-26]

K. Ueno, S. Nakamura, Tohoku Univ. (Japan); H. Shimotani, The Univ. of Tokyo (Japan);

T. Nojima, Tohoku Univ. (Japan); Y. Iwasa, The Univ. of Tokyo (Japan) and CREST, Japan

Science and Technology Agency (Japan); M. Kawasaki, Tohoku Univ. (Japan), The Univ. of Tokyo (Japan), and CREST, Japan Science and Technology Agency (Japan)

TOWARDS UNDERSTANDING OXIDES PROPERTIES III

7940 OR Valence structure of alkaline and post-transition metal oxides (Invited Paper) [7940-27]

J. A. McLeod, R. J. Green, Univ. of Saskatchewan (Canada); N. A. Skorikov, L. D. Finkelstein, Institute of Metal Physics (Russian Federation); M. Abu-Samak, Al-Hussein Bin Talal Univ. (Jordan); E. Z. Kurmaev, Institute of Metal Physics (Russian Federation); A. Moewes, Univ. of Saskatchewan (Canada)

7940 OT Cross-sectional scanning tunneling microscopy for complex oxide interfaces (Invited Paper) [7940-29]

T. Y. Chien, N. P. Guisinger, J. W. Freeland, Argonne National Lab. (United States)

NOVEL ZNO-BASED DEVICES/APPROACHES

$7940 \mathrm{OW}$ Enhanced output power using $\mathrm{MgZnO} / \mathrm{ZnO} / \mathrm{MgZnO}$ double heterostructure in $\mathrm{ZnO}$ homojunction light-emitting diode [7940-32]

S. Chu, Univ. of California, Riverside (United States), J. Zhao, Univ. of California, Riverside (United States) and Dalian Univ. of Technology (China), Z. Zuo, J. Kong, L. Li, J. Liu, Univ. of California, Riverside (United States)

7940 0X Application of dilute boron B(Al,In, Ga)N alloys for UV light sources (Invited Paper) [7940-58] S. Gautier, LMOPS, Univ. Paul Verlaine de Metz (France) and Supélec (France); M. Abid, GeorgiaTech-CNRS (France); T. Moudakir, Supélec (France); G. Orsal, LMOPS, Univ. Paul Verlaine de Metz (France) and Supélec (France); A. En Naciri, LMPD, Univ. Paul Verlaine de Metz (France); K. Pantzas, GeorgiaTech-CNRS (France); F. Jomard, LPSC, Univ. de Versailles Saint-Quentin (France); P. L. Voss, GeorgiaTech-CNRS (France); D. J. Rogers, F. H. Teherani, Nanovation (France); A. Ougazzaden, GeorgiaTech-CNRS (France)

$7940 \mathrm{OZ}$ Growth and characterization of ZnO nanostructures for UV sensor applications [7940-56] A. K. Sood, E. J. Egerton, Y. R. Puri, Magnolia Optical Technologies, Inc. (United States); J. Zeller, T. Manzur, Naval Undersea Warfare Ctr. (United States); D. L. Polla, N. K. Dhar, Defense Advanced Research Projects Agency (United States); J. Zhou, S. Xu, S. Zhang, Z. L. Wang, Georgia Institute of Technology (United States); A. F. M. Anwar, Univ. of Connecticut (United States)

\section{GROWTH AND PATTERNING OF OXIDES}

794011 Patterning of indium-tin-oxide (ITO) films using laser-induced forward transfer (LIFT) technique [7940-36]

H. Sakata, Tokai Univ. (Japan); A. Yoshikado, Zeta Photon Co., Ltd. (Japan); E. Yokoyama, M. Wakaki, Tokai Univ. (Japan) 
794012 Layer-by-layer epitaxial growth of polar MgO (111) films with atomically flat surfaces (Invited Paper) [7940-37]

K. Matsuzaki, H. Hosono, Tokyo Institute of Technology (Japan); T. Susaki, Tokyo Institute of Technology (Japan) and Japan Science and Technology Agency (Japan)

794013 Growth of metal oxide nanoparticles using pulsed laser ablation technique (Invited Paper) [7940-38]

M. A. Gondal, Q. A. Drmosh, T. A. Saleh, Z. H. Yamani, King Fahd Univ. of Petroleum and

Minerals (Saudi Arabia)

\section{OXIDE-BASED DEVICES}

794017 Pedestal anti-resonant reflecting optical waveguides [7940-42]

D. O. Carvalho, M. I. Alayo, Univ. de São Paulo (Brazil)

$79401 \mathrm{~A} \quad$ Hierarchical oxide-based composite nanostructures for energy, environmental, and sensing applications (Invited Paper) [7940-45]

P.-X. Gao, P. Shimpi, W. Cai, H. Gao, D. Jian, G. Wrobel, Univ. of Connecticut (United States)

POSTER SESSION

7940 1E Influence of Li implantation on the optical and electrical properties of ZnO film [7940-47] S. Nagar, B. Sinha, A. Mandal, Indian Institute of Technology Bombay (India); S. K. Gupta, Bhabha Atomic Research Ctr. (India); S. Chakrabarti, Indian Institute of Technology Bombay (India)

7940 IF Fabrication of ZnO channel waveguides for nonlinear optical applications [7940-48] E. Y. Morales Teraoka, T. Kita, Tohoku Univ. (Japan); A. Tsukazaki, The Univ. of Tokyo (Japan) and PRESTO, Japan Science and Technology Agency (Japan); M. Kawasaki, Tohoku Univ. (Japan) and CRESTO, Japan Science and Technology Agency (Japan); H. Yamada, TohokU Univ. (Japan)

$79401 \mathrm{G} \quad$ Nonlinear optical waveguides with rutile $\mathrm{TiO}_{2}$ [7940-49]

K. Abe, E. Y. Morales Teraoka, T. Kita, H. Yamada, Tohoku Univ. (Japan)

$79401 \mathrm{H} \quad$ Concentration dependent luminescence characteristics of ${ }^{5} \mathrm{D}_{4}$ and ${ }^{5} \mathrm{D}_{3}$ excited states of Tb ${ }^{3+}$ ions in CFB glasses [7940-50]

J. Suresh Kumar, K. Pavani, T. Sasikala, Sri Venkateswara Univ. (India); M. Jayasimhadri, Delhi Technological Univ. (India); K. Jang, Changwon National Univ. (Korea, Republic of);

L. Rama Moorthy, Sri Venkateswara Univ. (India)

794011 Integration of a micro-incandescent lamp and an interferometric filter for optical applications [7940-51]

H. Báez, M. I. Alayo, Univ. de São Paulo (Brazil) 
7940 1K Amorphous ZnO films grown by room temperature pulsed laser deposition on paper and mylar for transparent electronics applications [7940-53]

D. J. Rogers, Nanovation SARL (France); V. E. Sandana, Nanovation SARL (France), Northwestern Univ. (United States), and Ecole Polytechnique (France); F. H. Teherani, Nanovation SARL (France); R. McClintock, M. Razeghi, Northwestern Univ. (United States); H.-J. Drouhin, Ecole Polytechnique (France)

7940 IL ZnO ultraviolet photodetectors grown via thermal oxidation of $\mathrm{Zn}$-metal on glass and sapphire substrates [7940-54]

J. C. Moore, L. R. Covington, Coastal Carolina Univ. (United States); R. L. Foster, E. J. Gee, M. R. Jones, S. A. Morris, Longwood Univ. (United States)

Author Index 
Downloaded From: https://www.spiedigitallibrary.org/conference-proceedings-of-spie on 26 Apr 2023

Terms of Use: https://www.spiedigitallibrary.org/terms-of-use 


\title{
Conference Committee
}

\author{
Symposium Chair
}

Liang-Chy Chien, Kent State University (United States)

Symposium Cochairs

Klaus P. Streubel, OSRAM GmbH (Germany)

E. Fred Schubert, Rensselaer Polytechnic Institute (United States)

Program Track Chair

James G. Grote, Air Force Research Laboratory (United States)

Conference Chairs

Ferechteh Hosseini Teherani, Nanovation (France)

David C. Look, Wright State University (United States)

David J. Rogers, Nanovation (France)

Program Committee

Rodrigo Ferrão de Paiva Martins, CEMOP/Uninova (Portugal)

Elvira M. C. Fortunato, Universidade Nova de Lisboa (Portugal)

Michael D. Gerhold, U.S. Army Research Office (United States)

Hanns-Ulrich Habermeier, Max-Planck-Institut für Festkörperforschung (Germany)

Axel Hoffmann, Technische Universität Berlin (Germany)

Masashi Kawasaki, Tohoku University (Japan) and CREST, Japan

Science and Technology Agency (Japan)

Yicheng Lu, Rutgers, The State University of New Jersey (United States)

Tatsuo Okada, Kyushu University (Japan)

Manijeh Razeghi, Northwestern University (United States)

Zhong Lin Wang, Georgia Institute of Technology (United States)

Session Chairs

1 ZnO Doping I

David C. Look, Wright State University (United States)

Bruno K. Meyer, Justus-Liebig-Universität Giessen (Germany)

2 ZnO Doping II

David C. Look, Wright State University (United States)

Bruno K. Meyer, Justus-Liebig-Universität Giessen (Germany) 
$3 \quad$ ZnO Nanostructures: Growth and Device Fabrication I

Jean-Jacques Delaunay, The University of Tokyo (Japan)

Tatsuo Okada, Kyushu University (Japan)

$4 \quad$ ZnO Nanostructures: Growth and Device Fabrication II

Jean-Jacques Delaunay, The University of Tokyo (Japan)

Tatsuo Okada, Kyushu University (Japan)

5 Towards Understanding Oxides Properties I

Walter R. L. Lambrecht, Case Western Reserve University (United States)

6 Towards Understanding Oxides Properties II

Walter R. L. Lambrecht, Case Western Reserve University (United States)

7 Towards Understanding Oxides Properties III

Walter R. L. Lambrecht, Case Western Reserve University (United States)

8 Novel ZnO-based Devices/Approaches

David J. Rogers, Nanovation (France)

Aleksandra B. Djurišić, The University of Hong Kong (Hong Kong, China)

9 Growth and Patterning of Oxides I

Katharina Lorenz, Universidade de Lisboa (Portugal)

TeYu Chien, Argonne National Laboratory (United States)

10 Growth and Patterning of Oxides II

Katharina Lorenz, Universidade de Lisboa (Portugal)

TeYu Chien, Argonne National Laboratory (United States)

11 Oxide-based Devices I

Puxian Gao, Georgia Institute of Technology (United States)

Martin Allen, University of Canterbury (New Zealand)

12 Oxide-based Devices II

Puxian Gao, Georgia Institute of Technology (United States)

Martin Allen, University of Canterbury (New Zealand) 